\title{
Mucogingival Complications Following Orthodontic Space Closure; A Retrospective Study
}

\author{
Ahmad Bekdash ${ }^{2}$, Mohammed Faris ${ }^{2}$, Anas Allaham², Majd Eddin Alhorani² and Aous Dannan ${ }^{1 *}$ \\ ${ }^{1}$ Department of Periodontology, Faculty of Dentistry, The International University for Science and Technology (IUST), Syria \\ ${ }^{2}$ Department of Orthodontics, National Center of Dental Specialties and Syrian Board, Syria
}

*Corresponding author: Aous Dannan, Department of Periodontology, Faculty of Dentistry, The International University for Science and Technology (IUST), Damascus, Syria.

Received Date: August 03, 2020

Published Date: August 14, 2020

\section{Abstract}

Aim of the study: To investigate, retrospectively, the incidence of mucogingival complications after orthodontic space closure.

Materials and methods: A total of 35 files of patients, who were orthodontically treated in the Department of orthodontics at the National Center of Dental specialties and Syrian Board in Damascus/ Syria, were analyzed. Their treatment plans included orthodontic space closure. Patients were recalled and subjected to intra-oral examination to investigate cases of mucogingival complications. Chi Square statistical test was used to test the relationship between variables.

Results: No gingival complications were found in $32.3 \%$ of cases. Other complications varied among gingival inflammation, invaginations, clefts, and overgrowth. Gingival invaginations were found in $46.2 \%$ and $53.8 \%$ in the upper and lower jaws respectively. Gingival clefts were found in $61.5 \%$ and $38.5 \%$ in the upper and lower jaws respectively. No significant relationship was found between gingival complication and the method of orthodontic space closure.

Conclusion: Orthodontic space closure was not accompanied with high incidences of mucogingival complications (i.e. gingival invaginations and/or clefts). Most gingival complications were noted in the upper jaw compared to the lower jaw. No harmful features that could put the periodontal tissues at serious risk were documented.

Keywords: Gingival invagination; Cleft; Orthodontic; Space closure

\section{Introduction}

Several types of orthodontic treatments usually include extraction of specific teeth, most often premolars. Closure of orthodontic spaces is planned as a next step. Gingival invaginations (GIs) and gingival clefts (GCs) usually form during the orthodontic approximation of teeth $[1,2]$. If the formation and persistence of such an alteration in gingival contour predispose toward subsequent periodontal disease and relapse of tooth position, this would be inconsistent with the goals of orthodontic treatment [3]. The clinical appearance of such invaginations ranges from a minor one-surface crease in the attached gingiva to a deep cleft that extends across the interdental papilla from the buccal to the lingual alveolar surface.

The precise cause of these invaginations remains unclear. Since they occur more commonly in patients undergoing orthodontic closure of extraction spaces, they may be a consequence of the interruption of the continuity of the gingival fiber system and bone remodeling that occurs with destruction of cortical plates, socket healing, and root movement [4]. Alternatively, the gingival fiber system may be displaced rather than remodeled during tooth movement, the invagination forming as a result of a passive folding 
or pile-up of gingival tissue [5]. Alterations of the epithelium, connective tissue, and intercellular matrix have been demonstrated histologically [6]. The purpose of this investigation was to study, retrospectively, the incidence of mucogingival complications associated with orthodontic closure of extraction spaces in a sample of Syrian patients.

\section{Materials and Methods}

The records of 35 patients who underwent orthodontic space closure after different teeth extractions at the National Center for Dental Specializations (NDC) in Damascus, Syria, were randomly chosen for the study. All chosen cases were completed (i.e. extraction spaces were completely closed). Firstly, information regarding patient's gender, age, extracted teeth, the orthodontic method of space closure were collected and organized in a table to simplify data analysis. As a second step, all patients were contacted by the researchers and asked to visit the NDC clinics in order to undergo a recall oral examination. The existence of any case of gingival invaginations and/or clefts and/or gingival enlargements was documented, as well as the presence or absence of gingival inflammation on teeth that were orthodontically approximated (i.e. yes or no records). Statistical software program (SPSS, version 21) was used for data analysis. Chi-square test was used to find out the relationship among nominal variables.

\section{Results}

In the 35 patients (30 females "85\%" and 5 males "15\%) who were enrolled in this study, 119 teeth; namely upper and lower canines and premolars were investigated. According to the records reviewed, twenty teeth (i.e. canines and premolars) were orthodontically moved using the (power chain technique and lace back), and 99 teeth were orthodontically moved using the (loops). The canines and premolars were subjected to distal retraction forces according to every single group with the orthodontic techniques mentioned above.

The first premolars, both upper and lower, on both left and right sides, represented the most percentage of extracted teeth $(41.2 \%)$ (Figure 1). The (power chain and lace back) technique for orthodontic space closure was the most one used (82.4\%) (Figure 1).

The results showed no gingival complications in $32.4 \%$ of cases, and different ones in the rest of cases with gingival enlargement as the highest percentage (11.8\%). Most gingival complications were noted in the upper jaw (23.5\%) compared to the lower jaw (8.8\%). The rest of complications were distributed in a fairly compatible way between both jaws, also on both left and right sides (Figure $2,3)$.

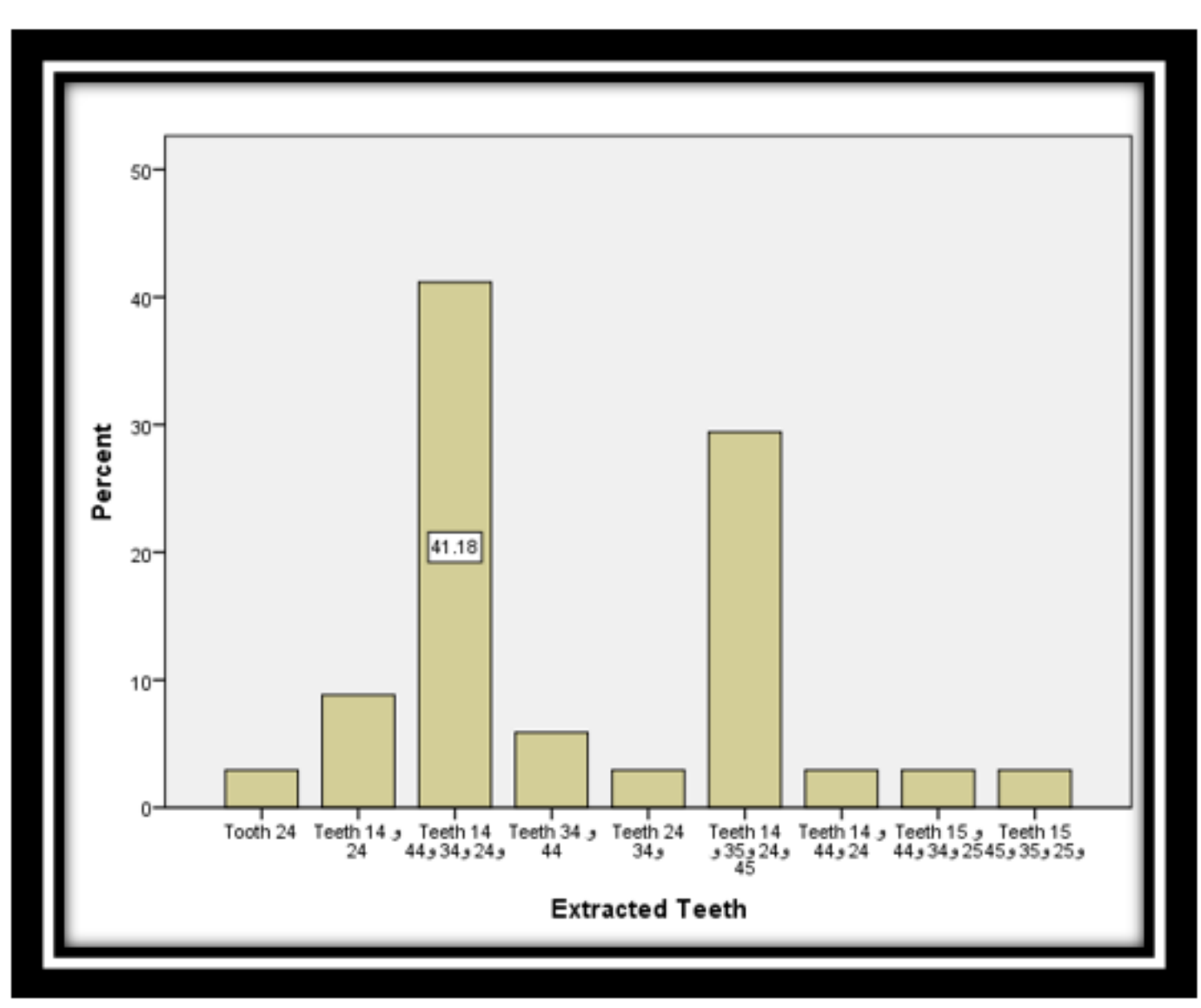

Figure 1: Distribution of extracted teeth prior to active orthodontic treatment. 


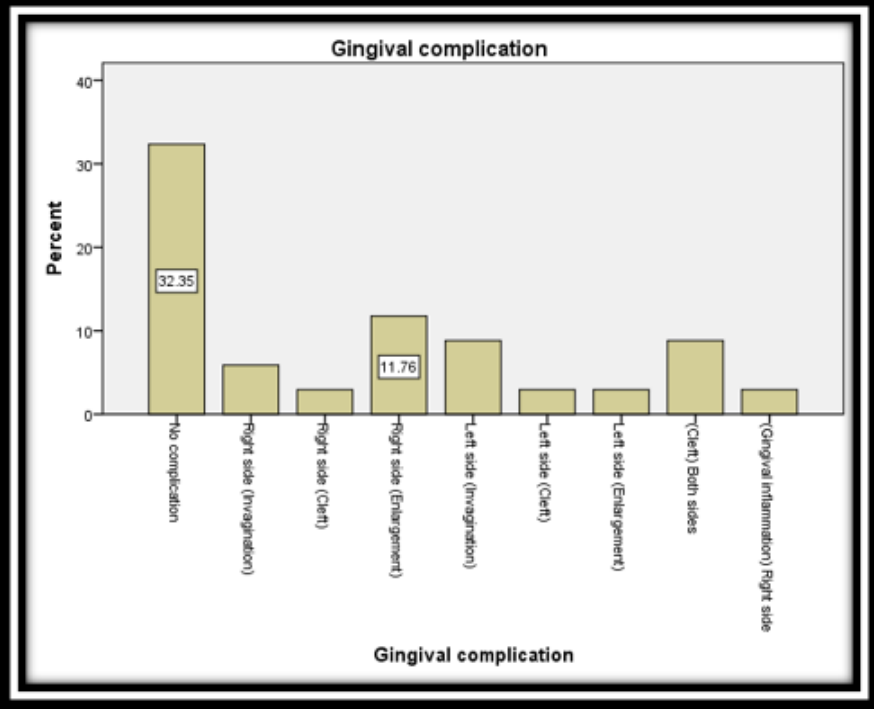

Figure 2: Mucogingival complications due to orthodontic space closure.

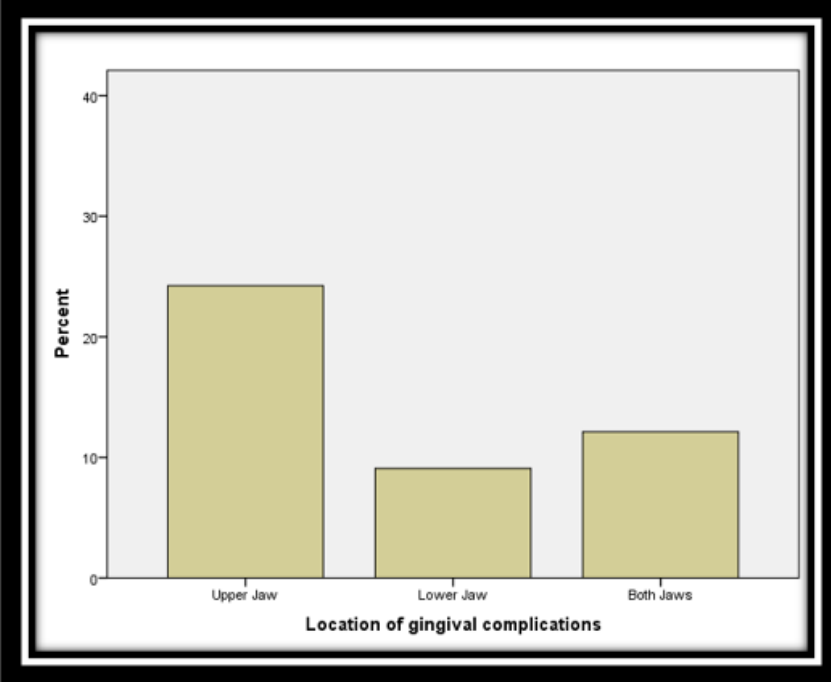

Figure 3: Location of gingival complications between jaws.

Gingival invaginations were found in $46.2 \%$ and $53.8 \%$ in the upper and lower jaws respectively. Gingival clefts were found in $61.5 \%$ and $38.5 \%$ in the upper and lower jaws respectively (Table $1)$.

Table 1: Distribution of gingival invaginations and clefts between jaws.

\begin{tabular}{|c|c|c|c|c|c|c|}
\hline \multicolumn{7}{|c|}{ Crosstabulation } \\
\hline & & & \multicolumn{3}{|c|}{ Gingival complication } & \multirow{2}{*}{ Total } \\
\hline \multicolumn{3}{|c|}{ Gingival invagination Gingival cleft } & Gingival enlargement & & & \\
\hline \multirow{6}{*}{ Jaw } & \multirow{3}{*}{ Upper jaw } & Count & 6 & 8 & 5 & 19 \\
\hline & & Percentage within (jaws) & $31.60 \%$ & $42.10 \%$ & $26.30 \%$ & $100.00 \%$ \\
\hline & & Percentage within (gingival complication) & $46.20 \%$ & $61.50 \%$ & $45.50 \%$ & $51.40 \%$ \\
\hline & \multirow{3}{*}{ Lower jaw } & Count & 7 & 5 & 6 & 18 \\
\hline & & Percentage within (jaws) & $38.90 \%$ & $27.80 \%$ & $33.30 \%$ & $100.00 \%$ \\
\hline & & Percentage within (gingival complication) & $53.80 \%$ & $38.50 \%$ & $54.50 \%$ & $48.60 \%$ \\
\hline
\end{tabular}


Table 2: Analysis of relationship between gender and gingival complication.

\begin{tabular}{|c|c|c|c|c|}
\hline \multicolumn{5}{|c|}{ Chi-Square Test } \\
\hline & Value & Degrees of freedom & P Value & Significance \\
\hline Chi-Square & 13.707 & 14 & 0.47 & Not significant \\
\hline
\end{tabular}

Table 3: Analysis of relationship between orthodontic method and gingival complication.

\begin{tabular}{|c|c|c|c|c|}
\hline \multicolumn{5}{|c|}{ Chi-Square Test } \\
\hline & Value & Degrees of freedom & P Value & Significance \\
\hline Chi-Square & 13.468 & 28 & 0.99 & Not significant \\
\hline
\end{tabular}

\section{Discussion}

Periodontal health is directly related to the accumulation of bacterial plaque [7], the primary etiologic agent in gingivitis and periodontitis. Factors that predispose to more plaque accumulation may increase the risk of marginal gingivitis. This relatively mild form of periodontal disease is generally considered to be reversible, but can, in time, progress to destructive periodontal disease if the accumulated plaque is not removed. Gingival invaginations may persist for as long as 5 years after completion of orthodontic treatment [2]. If gingival invaginations predispose, either directly or indirectly through poor interproximal contacts, to plaque accumulation and hence gingivitis, their formation during and persistence after orthodontic tooth movement could have an adverse effect on future periodontal health.

In a recent study, Bertel $\mathrm{K}$, et al. [8] found that the incidence of gingival clefts is higher in cases where orthodontic space closure had been initiated early and in fast rate [8]. The current study did not show any relationship between gender of patients and the nature of mucogingival complication. This might give a sign that the mechanism of such complications is not affected by the hormonal nature of the host (i.e. between males and females). Similarly, no relationship was found between the method used for space closure and the mucogingival complication. However, most of gingival deformities were noted closely to the power chain technique and lace back.

\section{Conclusion}

According to this retrospective study, orthodontic space closure by means of power chain technique and lace back as well as the loops technique was not accompanied by higher incidence of mucogingival complications. This study could have been more reliable if the effect of patients' oral hygiene had been studied. However, such a variable is usually hard to be controlled in retrospective studies. More prospective studies in this regard is still needed.

\section{Acknowledgment}

The authors are very thankful to Prof Dr Fadia Dib, Head director of NDC, for the facilities she offered in order to make this research more valuable. Special thanks also to Dr Naji Masoud and Dr Muhannad Kazzaz for their precious advice regarding the structure of the research.

\section{Conflict of Interest}

No conflict of interest.

\section{References}

1. Gölz L, Reichert C, Jäger A (2011) Gingival invagination-a systematic review. J Orofac Orthop 72: 409-420.

2. Reichert C, Gölz L, Dirk C (212) Retrospective investigation of gingival invaginations: Part I: clinical findings and presentation of a coding system. J Orofac Orthop 73: 307-316.

3. Rivera Circuns AL, Tulloch JF (1983) Gingival invagination in extraction sites of orthodontic patients: their incidence, effects on periodontal health, and orthodontic treatment. Am J Orthod 83: 469-476.

4. Robertson PB, Schultz LD, Levy BM (1977) Occurrence and distribution of interdental gingival clefts following orthodontic movement into bicuspid extraction sites. J Periodontol 48: 232-235.

5. Gölz L, Reichert C, Dirk C (2012) Retrospective investigation of gingival invaginations: Part II: microbiological findings and genetic risk profile. J Orofac Orthop 73: 387-396.

6. Diedrich P, Wehrbein H (1997) Orthodontic retraction into recent and healed extraction sites. A histologic study. J Orofac Orthop 58: 90-99.

7. Silness J, Loe H (1964) Periodontal disease in pregnancy. II. Correlation between oral hygiene and periodontal condtion. Acta Odontol Scand 22: 121-135.

8. Bertl K, Neuner H, Meran A, Michael H Bertl, Ilse Reich, et al. (2019) Does the time-point of orthodontic space closure initiation after tooth extraction affect the incidence of gingival cleft development? A randomized controlled clinical trial. J Periodontol 91(5): 572-581. 